\title{
Understanding and the Norm of Explanation*
}

\author{
John Turri \\ john.turri@gmail.com
}

Abstract: I propose and defend the hypothesis that understanding is the norm of (the speech act of) explanation. On this proposal, an explanation should express understanding. I call this the understanding account of explanation. The understanding account is supported by social and introspective observations. It is also supported by the relationship between knowledge and understanding, on the one hand, and assertion and explanation, on the other.

keywords: understanding; explanation; norms; knowledge; assertion

word count: 1690

It is widely accepted that understanding is closely related to explanation (e.g. Aristotle, 350 BCE; Kim, 1999; Lipton, 2004). While many philosophers have offered theories about the relationship (e.g. Salmon, 1989; Grimm, 2010; Strevens, 2013), one attractive possibility has not been explicitly identified and developed. The point of this paper is to identify and begin developing this possibility.

I propose that one deep and important aspect of the relationship is normative. More specifically, I propose that understanding is the norm of explanation. On this proposal, an explanation should express understanding. Call this the understanding account of explanation.

* This is the penultimate version of a paper forthcoming in Philosophia. Please cite the final, published version if possible. 
In one sense of "explanation," one fact or event explains another by causing or otherwise producing it. But this is not the sense most relevant to the understanding account. Instead, the understanding account focuses on the speech act of explanation - a linguistic performance consisting of one or more assertions that answer questions about the thing being explained. I do not deny that the different senses of "explanation" or their referents are related in important ways. Nevertheless, my focus is the speech act.

The understanding account is supported by social observation and introspection. More specifically, it is supported by patterns surrounding the ordinary give-and-take of explanation. There are at least six such patterns.

First, questions about understanding can function as indirect requests to provide explanations. That is, we can effectively prompt explanations by asking about understanding. For example, the question "Do you understand why/how this fire started?" is naturally understood as a request for explanation, and the response "Sure, let me explain ..." is fully competent and attentive. But why would that be, if an explanation is not requested in the literal content of the question? If understanding is the norm of explanation, then the question "Do you understand why/ how this happened?" enables us to infer that this person wants us to explain why/how it happened and, thus, can function as an indirect request for an explanation. This is similar to the way one's question to a bureaucrat, "Are you authorized to make an exception in this case?" can serve as an indirect request for the bureaucrat to show mercy and make an exception. Notice, furthermore, that in the cases of the fire and the bureaucrat, it is not incompetent to response by saying "Yes I do, but I will not explain it to you" or "Yes I am authorized, but I will not make an excep- 
tion in your case." Such responses might be rude but they wouldn't exhibit misunderstanding of what such questions imply.

Second, we can appropriately abstain from offering explanations by citing lack of understanding. Suppose the topic of conversation is the recent fire and you're asked, "How did this happen?" It is perfectly acceptable to respond, "Sorry, I don't understand it myself." But you and what you understand are irrelevant to the content of the question, so why is that response any more acceptable than, say, "Sorry, I get depressed when fires occur." If understanding is the norm of explanation, then by saying "I don't understand," you inform the questioner that you lack the authority to offer an explanation, which is surely relevant in the context.

Third, questions and remarks about understanding are appropriate in light of an offer to explain events. For example, suppose someone offers to explain why the fire occurred, "Let me tell you why this happened." It is appropriate to respond, "You understand why it happened?" or, “Oh, good, I'm glad someone here understands why it happened." Why are such questions and inferences sensible? If understanding is the norm of explanation, then by offering to explain, you represent yourself as satisfying the norm, namely, as understanding. And by representing yourself this way, you make such questions and inferences sensible.

Fourth, more aggressive than "You understand why it happened?" is "But you don't understand why it happened." What explains this ordering of aggressiveness? If understanding is the norm of explanation, we can explain it as follows. "You understand why it happened?" can be used to challenge your authority to provide an explanation by questioning whether you have it, whereas "But you don't understand why it happened" explicitly rejects your authority to provide 
an explanation. Explicitly rejecting someone's authority is more aggressive than merely questioning whether someone has authority.

Fifth, citing your understanding vindicates an explanation that is accused of illegitimacy. Suppose the question arises, "Why did the fire occur?" and you offer an explanation. Someone levels the accusation, "You're not in a position to explain this event." Responding with, "Yes I am - I understand why it happened," would, if true, fully vindicate the explanation. Indeed, your response seems to flatly contradict the accusation. If understanding is the norm of explanation, this is easily explained. How obtuse your accuser would seem if he answered that your response had missed the point. (Accusations made on ethical or legal grounds are different and would have to be handled differently. Such accusations are also irrelevant to my discussion here.)

Sixth, certain offers strike us as inconsistent. For example, it sounds absurd to say, "I don't understand why it happened, but I can explain why it happened," or, "I don't understand how it happened, but here is how it happened ...”. Why do such offers seem inconsistent? If understanding is the norm of explanation, then by making the offer you represent yourself as understanding. But in the same breath you claim that you don't understand. Thus, the inconsistency results from explicitly saying that you lack the authority which you represent yourself as having.

If understanding is the norm of explanation, then we can explain all six observations in a simple, elegant, and unified way. This is good initial evidence for the hypothesis that understanding is the norm of explanation.

The understanding account is also supported by an argument from three independently plausible premises. First, knowledge is the norm of assertion (MacIver, 1938; Unger, 1975; 
Williamson, 2000; Turri, 2010; Benton, 2011; Turri 2013; Turri 2014; Turri 2015a; Turri 2015b; Turri in press a; Turri in press b; for a literature review, see Benton, 2014). Second, an explanation consists of one or more assertions answering questions about a fact or event's occurrence, such as "why?" and "how?". (Note that this simply reiterates the conception of the speech act under investigation here.) Third, understanding consists in knowing the answer to such questions (Achinstein, 1983; Lipton, 2004; Grimm, 2006). If these three premises are correct, then the understanding account of explanation is just a special instance of the knowledge account of assertion: explanation is a special form of assertion, and understanding is the corresponding special form of knowledge.

I suspect that the understanding account is often just below the surface in many discussions of understanding and explanation, even if no one has explicitly stated and defended it. One esteemed philosopher of science defines "explanation" as "uttering something with the intention of rendering [a fact or event] understandable" (Achinstein, 1983, p. 23). In a textbook treatment of Carl Hempel's enormously influential theory of explanation, another philosopher writes in passing, "Explanation has to do with understanding. So an adequate explanation of [an event] should offer an adequate understanding" of the event (Psillos, 2002, p. 218). Passages like these suggest that philosophers of science have recognized, at least implicitly, the attractiveness of the understanding account. The present discussion advances our understanding of these issues by explicitly proposing the understanding account, marshaling observational evidence on its behalf, and connecting it to related issues in epistemology and the philosophy of language.

According to my proposal, the content of the norm is that an explanation should express 
understanding. For instance, if someone asks, "Why did the fire occur?" and you respond, "Because of a short circuit," then your statement should express understanding, which in this case amounts to expressing knowledge that the short circuit caused the fire. My proposal, while novel and informative, leaves open some important questions for future work to investigate. For instance, none of the evidence reviewed here seems to require that the norm be understood as imposing a strict necessary or sufficient condition, as opposed to, say, a very strong central tendency that allows exceptions (for further discussion, see Turri in press c). Similarly, current evidence does not require a specific account of the normative status expressed by "should." For instance, it could pertain to when an explanation is permissible, or it could pertain to when an explanation is good, or it might be irreducible to these or any other. Relatedly, because existing evidence makes it likely that the speech act of assertion is constituted by a knowledge norm (see Turri in press a), if my reasoning above is correct, then it is also likely that the speech act of explanation is constituted by an understanding norm. However, caution is warranted on this point. According to the best available evidence, the constitution of the human practice of assertion is a special case of the constitution of animal signaling practices more generally (see Turri in press a). But even if an explanation is just a special form of assertion, it still is special, communicating not merely that something is true but also why or how. And it is far from clear that communicating why or how is a typical feature of animal communication systems. So matters are likely to be more complicated for explanation specifically. One possibility is that information about rationale or mechanism is crucial to the development, acquisition, and maintenance of complex social and material culture, traits common among primates and developed to their greatest extent among humans (for a re- 
view, see Whiten, 2011).

It would be counterproductive to claim false precision on any of these points at this stage of inquiry. Some of the recent literature on norms of assertion suffers from precisely this defect, with many debates focusing on extremely detailed proposals before enough evidence existed to responsibly evaluate them (for further discussion of this point, see Turri in press a). A more productive strategy is to seek further evidence enabling us to sharpen our understanding of the norm's content, status, and function. As has been the case with investigation of the norm of assertion and other speech acts, this will likely involve a combination of further social observation, behavioral experimentation, and input from the social, cognitive, and life sciences.

Acknowledgments - For feedback I thank Matthew Benton, Stephen Grimm, and Angelo Turri. This research was supported by the Social Sciences and Humanities Research Council of Canada and an Early Researcher Award from the Ontario Ministry of Economic Development and Innovation.

\section{References}

Achinstein, P. (1983). The nature of explanation. Oxford: Oxford University Press.

Aristotle. (350 BCE/1941). Posterior analytics. In R. McKeon (Ed.), G. R. G. Mure (Trans.), The basic works of Aristotle. New York: Random House.

Benton, M. A. (2011). Two more for the knowledge account of assertion. Analysis, 71(4), 684- 
687. doi:10.1093/analys/anr085

Benton, M. A. (2014). Knowledge norms. Internet Encyclopedia of Philosophy. Retrieved January 2014, from http://www.iep.utm.edu/kn-norms/.

Grimm, S. R. (2006). Is Understanding A Species Of Knowledge? The British Journal for the Philosophy of Science, 57(3), 515-535. doi:10.1093/bjps/ax1015

Grimm, S. R. (2010). The goal of explanation. Studies in History and Philosophy of Science, 41(4), 337-344. doi:10.1016/j.shpsa.2010.10.006

Kim, J. (1999). Hempel, explanation, metaphysics. Philosophical Studies, 94(1), 1-20.

Lipton, P. (2004). Inference to the best explanation (2nd ed.). new York: Routledge.

MacIver, A. M. (1938). Some questions about 'know' and “think.” Analysis, 5(3), 43-50.

Psillos, S. (2002). Causation \& Explanation. Montreal: McGill-Queen's.

Salmon, W. C. (n.d.). Four decades of scientific explanation. In P. Kitcher \& W. C. Salmon (Eds.), Minnesota studies in the philosophy of science (Vol. 13). Minneapolis: University of Minnesota Press.

Strevens, M. (2013). Studies in History and Philosophy of Science. Studies in History and Philosophy of Science, 44(3), 510-515. doi:10.1016/j.shpsa.2012.12.005

Turri, J. (2010). Prompting challenges. Analysis, 70(3), 456-462. doi:10.1093/analys/anq027

Turri, J. (2013). The test of truth: An experimental investigation of the norm of assertion. Cognition, 129(2), 279-291. http://doi.org/10.1016/j.cognition.2013.06.012

Turri, J. (2014). Knowledge and suberogatory assertion. Philosophical Studies, 167(3), 557-567. http://doi.org/10.1007/s11098-013-0112-z 
Turri, J. (2015a). Knowledge and the norm of assertion: a simple test. Synthese, 192(2), 385392. http://doi.org/10.1007/s11229-014-0573-4

Turri, J. (2015b). Selfless assertions: some empirical evidence. Synthese, 192(4), 1221-1223. http://doi.org/10.1007/s11229-014-0621-0

Turri, J. (in press a). Knowledge and the norm of assertion: an essay in philosophical science. Open Book Publishers.

Turri, J. (in press b). Knowledge, certainty and assertion. Philosophical Psychology.

Turri, J. (in press c). Sustaining rules: a model and application. In J. A. Carter, E. C. Gordon, \& B. Jarvis (Eds.), Knowledge first. Oxford University Press.

Unger, P. (1975). Ignorance: a case for skepticism. Oxford: Oxford University Press.

Whiten, A. (2011). Social learning, traditions, and culture. In J. C. Mitani, J. Call, P. M. Kappeler, R. A. Palombit, \& J. B. Silk (Eds.), The evolution of primate societies (pp. 682-700). University of Chicago Press.

Williamson, T. (2000). Knowledge and its limits. Oxford: Oxford University Press. 